\title{
Photoreaction of New Chalcone-Based Side Chain Type Liquid Crystalline Polymers and Alignment Behavior of Liquid Crystal on the Irradiated Films
}

\author{
Takashi Minara, Machiko Tsutsumi, and Naoyuki KoIDE ${ }^{\dagger}$ \\ Department of Chemistry, Faculty of Science, Science University of Tokyo, 1-3 Kagurazaka, \\ Shinjuku-ku, Tokyo 162-8601 Japan
}

(Received November 19, 2001; Accepted March 12, 2002)

\begin{abstract}
We investigated the photoreaction of the side chain type liquid crystalline polymers with a chalcone derivative in the side chain. The polymer films were irradiated with linearly polarized UV (LPUV) light (350 nm) under different conditions. The photodimerization of the chalconyl moieties would mainly occur due to a decrease in the peak assigned to the chalconyl moiety by UV-vis spectroscopy measurements. The extent of the photoreaction in the liquid crystalline (LC) state is larger than that in the solid state after spin-coating and in the locked-in LC state after annealing. The LC cell was fabricated from the polymer film irradiated with LPUV light to examine the alignment properties of low molar mass LC molecules on them. A mixture of low molecular liquid crystal with the dichroic dye was filled in the LC cell. The low molecular liquid crystal was easily aligned parallel to the electric vector of the incident exposed LPUV light in the LC cell with the polymer film irradiated in the LC state.

KEY WORDS Chalcone / Liquid Crystalline Polymer / Photodimerization / Photoalignment /
\end{abstract}

The alignment of liquid crystalline (LC) molecules plays an important role for liquid crystalline displays (LCDs). The rubbing process has been mainly employed for the uniaxial orientation of the LC molecules. However, the rubbing process has many disadvantages for LCDs such as generation of electrostatic charge and dust. Therefore, many methods of alignment technique for LC molecules are focused on rubbing free methods. Photoalignment procedure is recognized as one of rubbing free methods and is investigated as one of candidates that overcome the generation of electrostatic charge and dust for the rubbing process. ${ }^{1,2}$

Photoalignment procedure is based on photochemical reactions such as photoisomerization, photodimerization (photocrosslinking) and photodecomposition. With respect to photoisomerization, polymers containing an azobenzene derivative were intensively investigated. ${ }^{3-5}$ The reversible photoreaction of the azobenzene moieties in the polymer induced a reversible change in the alignment direction of low molecular weight LC molecules. Generally photodimerization of photosensitive groups in the polymer was employed to obtain a stable alignment of low molecular weight LC molecules.

Photodimerization of the cinnamoyl chromophore in the polymer film have been studied in detail. The polymer films containing dimerized moiety of cinnamoyl group have been investigated as a photoalignment material by many researches. ${ }^{2,6-10}$ Schadt et al. have ob- tained the alignment film by photodimerization of cinnamoyl group in the poly(vinyl 4-methoxycinnamate). LC molecules in the LC cell were aligned perpendicular to the electric vector of the incident LPUV light. ${ }^{6}$

Alignment behavior of LC molecules would be easily improved by the liquid crystalline polymer surface where mesogenic groups are aligned. Kawatsuki et al. had reported alignment properties of side chain type liquid crystalline polymers with the cinnamoyl groups as an alignment film. ${ }^{11,12}$

It would be desirable that photodimerization occurs by the exposure of relatively longer wavelength of the UV light to avoid partial degradation of polymer films by the irradiation of the UV light. ${ }^{13}$ It is well known that the photoreaction of chalconyl moieties takes place by irradiation with relatively longer wavelength of the UV light. Photosensitive polymers containing chalcone derivatives have been studied for the photoalignment film. ${ }^{13-19}$ Makita et al. reported that the good LC alignment was observed for the LC cell using the polymer film with a chalcone moiety compared to the cinnamate polymer film when the deep UV light was cut off. ${ }^{13-16}$ So the chalconyl moiety is a good photosensitive group. Poly(4-methacryloyloxy chalcone) (PM4Ch) or poly (4'-methacryloyloxy chalcone) (PMCh) were employed as a photoalignment film. The LC molecules in the LC cells using the linearly polarized UV (LPUV) irradiated PM4Ch or PMCh film were aligned to perpendicular to the electric vector of the in-

${ }^{\dagger}$ To whom correspondence should be addressed (Tel: +81-3-3260-4272 ext. 2262, Fax: +81-3-3235-2214, E-mail: nkoide@ch. kagu.sut.ac.jp). 


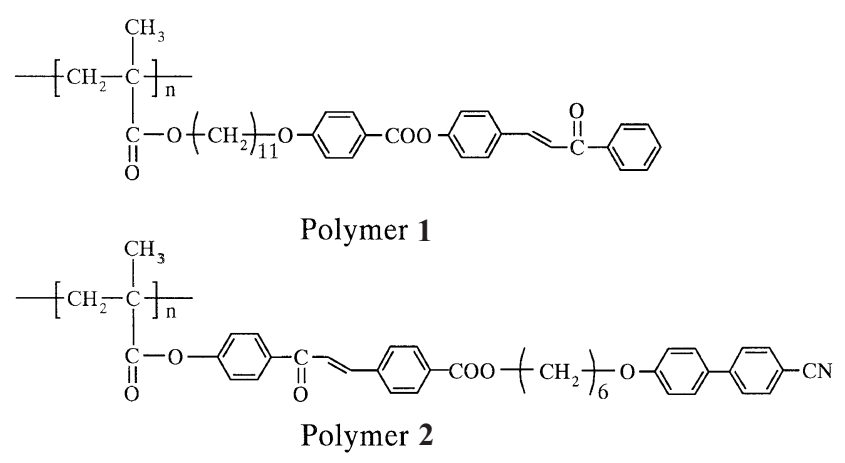

Figure 1. Chemical structures of side chain type polymers.

cident LPUV light. ${ }^{13,14}$ In the case of the photoalignment films on which LC molecules were aligned perpendicular to the electric vector of the incident LPUV light, the generation of pretilt angle would require the two step LPUV irradiation as suggested by Iimura et $a .^{20}$

We thought that the alignment behavior of the LC molecules would be easily improved by the liquid crystalline polymer surface where mesogenic groups having similar chemical structure to that of the LC molecules existed. In this study, we investigated the photoreaction of the chalcone-based LC polymers films. The extent of the photoreaction for the chalconyl group in the LC polymers was examined by UV-vis spectroscopy measurements. The LC cell was fabricated from the polymer film irradiated with the LPUV light. A mixture of low molecular liquid crystal with the dichroic dye was filled in the LC cell. The alignment direction of the LC molecules was discussed by dichroic ratio of the dichroic dye in the LC cell.

\section{EXPERIMENTAL}

\section{Materials}

Chemical structures of the LC polymers were shown in Figure 1. Synthesis and thermal properties of polymers $\mathbf{1}$ and $\mathbf{2}$ were described in the literature. ${ }^{21}$

\section{Characterization}

UV-vis spectroscopy measurements were carried out with a HITACHI U-3410 spectrophotometer. FT-IR measurements were carried out using JEOL JIR-7000 with infrared microscope unit.

\section{Linearly Polarized UV Irradiation}

The polymer film was made by spin-coating on a quartz substrate from a chloroform solution. The thickness of the polymer 1 film was about $100 \AA$, while the thickness of the polymer 2 film was about $160 \AA$. The polymer film was irradiated with a $500 \mathrm{~W}$ super highpressure mercury lamp with a glan laser prism and a cut
Table I. Thermal properties of side chain type polymers containing chalcone moiety

\begin{tabular}{|c|c|c|c|c|c|}
\hline Polymers & \multicolumn{5}{|c|}{ Phase transition temperatures $/{ }^{\circ} \mathrm{C}$} \\
\hline \multirow{2}{*}{ Polymer 1} & & 34 & \multirow{2}{*}{ Sm } & 196 & \\
\hline & $\mathrm{g}$ & 31 & & 188 & \\
\hline \multirow{2}{*}{ Polymer 2} & \multirow{2}{*}{$\mathrm{g}$} & 87 & \multirow{2}{*}{$\mathrm{Sm}$} & 165 & \\
\hline & & 78 & & 162 & 1 \\
\hline
\end{tabular}

g; glassy, Sm; smectic, I; isotropic.

filter $(350 \mathrm{~nm})$. The LPUV light intensity was $1.89 \mathrm{~mW}$ $\mathrm{cm}^{-2}$ at $365 \mathrm{~nm}$. The polymer film was irradiated with the LPUV light under different conditions as follows: (1) irradiation at room temperature after spin-coating, (2) irradiation at room temperature after annealing the spin-coated film in a LC state, (3) irradiation in a LC state.

\section{Alignment of LC Molecules}

A sandwich type LC cell was fabricated in an antiparallel fashion of the polymer films irradiated with the LPUV light. A mixture of the low molecular weight liquid crystal (MBBA; $p$-methoxybenzylidene$p$ - $n$-butylaniline) with the dichroic dye (M137) was injected in the LC cell. The thickness of the LC cell was $10 \mu \mathrm{m}$. Alignment of the LC molecules was investigated based on the dichroic ratio $(D R)$ of the absorbance at $640 \mathrm{~nm}$ for the dichroic dye, which were obtained by polarized UV-vis spectroscopy measurements. The $D R$ was defined as follow:

$$
\text { Dichroic ratio }(D R)=\frac{A_{\text {para }}-A_{\text {per }}}{A_{\text {para }}+A_{\text {per }}}
$$

$A_{\text {para }}$ and $A_{\text {per }}$ indicate the absorbance parallel and perpendicular to the incident electric vector of the exposed LPUV light, respectively.

\section{RESULTS AND DISCUSSION}

\section{Thermal Properties of Polymers}

We synthesized two types of polymers containing chalconyl moieties, one is a polymer having the chalconyl moiety directly connected to the benzoate component, and the other is a polymer with a spacer group introduced between the cyanobiphenyl and the chalconyl moiety. Synthesis of these monomers and polymers were described in other literature. ${ }^{21}$ The numberaverage molecular weight of the polymer 1 was 27300 and the dispersion of the polymer 1 was 3.7, while that of the polymer 2 was 3300 and the dispersion was 1.2.

Thermal properties of the polymers were summarized in Table I. A typical optical texture was not observed for both types of polymers by polarized optical microscopy measurements, however a birefringence 

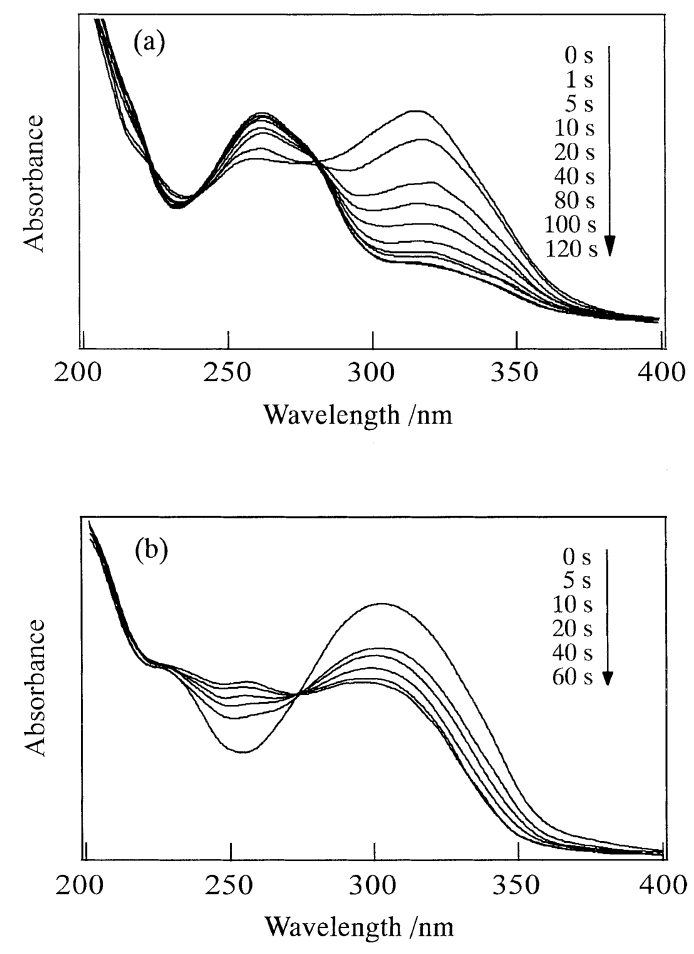

Figure 2. Absorption spectral changes of polymers exposed to linearly polarized UV light in the liquid crystalline state: (a) polymer 1 irradiated at $170^{\circ} \mathrm{C}$ and (b) polymer 2 irradiated at $150^{\circ} \mathrm{C}$.

was shown for these polymers. A shift of a baseline and a peak in DSC curves of polymer 1 were observed on the both heating and cooling scans. Similar DSC curves were detected by DSC measurements of the polymer 2 . The shift of the baseline was attributed to glass transition, while the peak was assigned to the transition from the mesophase to the isotropic melt. The clearing point of these polymers was confirmed by polarized optical microscopy measurements. A birefringence of the polymers disappeared at the clearing point. A mesophase of both polymers was exhibited in the temperature range between the glass transition and the clearing point. The optical texture of both polymers was not altered by the phase transition from the mesophase to glassy state. This result indicated that the mesophase structure of the polymers would be maintained below the glass transition.

The details of the mesophase structure were investigated by X-Ray measurements. Two sharp peaks in the small-angle region and a broad peak in the wideangle region were observed in the X-Ray pattern of the polymer 1. We obtained $40 \AA$ of $\mathrm{d}$-spacing due to the first sharp peak in the small-angle region for the polymer 1. The calculated length for the side chain with an extended conformation of the polymer 1 was $37 \AA$. Similar X-Ray patterns were detected for the polymer 2. We concluded that the polymers $\mathbf{1}$ and $\mathbf{2}$ exhibited a smectic $\mathrm{A}_{1}$ phase.

\section{Photoreaction of Polymer Films}

UV-vis spectra of the polymer film exposed to the LPUV light in the LC state were shown in Figure 2. The absorption spectra of the polymer were obtained by UV-vis spectroscopy measurements after irradiation with the LPUV light. A peak around $320 \mathrm{~nm}$ in the spectra of the polymer $1 \mathrm{film}$ as shown in Figure 2a, decreased with increasing irradiation time, while a peak around $260 \mathrm{~nm}$ increased with increasing irradiation time.

The same tendency of the changes in the UV-vis spectra was observed for the polymer 2 film. The peak around $300 \mathrm{~nm}$ of the polymer 2 film was attributed to the absorption of the trans chalcone derivative and the cyanobiphenyl group. Therefore a small decrease in the absorption of the peak around $300 \mathrm{~nm}$ was observed compared to a decrease in the absorption peak around $320 \mathrm{~nm}$ for the polymer $\mathbf{1}$ film. The peak around $320 \mathrm{~nm}$ was assigned to the mesogenic group containing the chalcone derivative with the trans conformation, while the peak around $260 \mathrm{~nm}$ in the spectra of the polymer 1 film was attributed to the dimer structure formed by the photoreaction. ${ }^{14}$

The photodimerization and photoisomerization are known as a possible photoreaction of the photoreactive molecules like the chalconyl or the cinnamoyl groups. We investigated the photoreaction of the chalconyl groups by FT-IR measurements. We confirmed that a main photoreaction of the chalconyl groups in our polymer films was attributed to the photodimerization on the basis of a decrease in the peak $\left(984 \mathrm{~cm}^{-1}\right)$ assigned to the olefin $\mathrm{C}-\mathrm{H}$ out-of-plane bending for the trans chalconyl groups and a shift to the higher wave number of the peak $\left(1666 \mathrm{~cm}^{-1}\right)$ assigned to the ketone group. ${ }^{13}$

Figure 3 showed the extent of photoreaction for the polymers. The extent of the photoreaction was estimated by the decrease in the absorption peak around $320 \mathrm{~nm}$. The extent of the photoreaction in the LC state was larger than that in other states (as described in experimental section, spin-coated film (1) and annealed film (2)). Furthermore, the photoreaction of the polymer films in the LC state was faster than that of the polymer films in other states. These results indicated that the extent of photodimerization would be dependent upon the orientation and thermal motion of the chalcone moiety.

In the annealed film, the orientation of the chalcone moiety would be similar to that in the LC state, because LCPs can lock-in the mesogenic orientational order below the glass transition temperature. If only the orientation of the chalcone moiety were important for the extent of the photodimerization, the extent of the pho- 

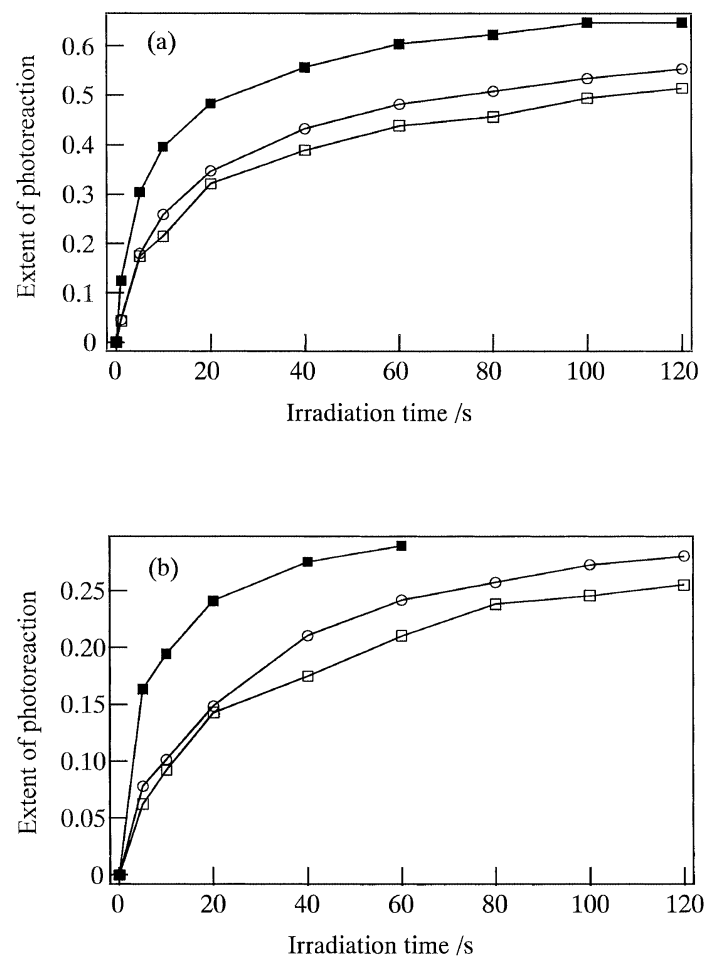

Figure 3. The extent of photoreaction of polymer 1 (a) and polymer 2 (b) films: the polymer films irradiated with linearly polarized UV light in the LC state (filled squares), after annealing (open squares) and after spin-coating (open circles).

todimerization in the annealed film should be larger as well as that in the LC state. However the extent of the photodimerization in the annealed film was not so large compared to that in the other states. The result indicated that its thermal motion of the chalcone moiety in the LC state would play an important role in larger extent of photodimerization.

Thermal motion of the chalcone moiety in the LC state would occur when the polymer film was heated above its glass transition temperature. Therefore the photodimerization temperature would be one of the important factors in determining the extent of the photodimerization. In this study, we employed the reduced temperature $(0.86)$ for the photodimerization temperature in the LC state, however appropriate temperature condition for the photodimerization of the chalcone moiety would be present.

\section{Photoinduced Anisotropy of Polymer Films}

We measured the dichroic ratio $(D R)$ of the polymer 1 film irradiated with the LPUV light to investigate the photoinduced anisotropy of the film. Figure 4 showed $D R$ values of the peaks at $260 \mathrm{~nm}$ and $320 \mathrm{~nm}$ in the polarized UV spectra of the polymer 1 films irradiated with the LPUV light under three different conditions. The peak at $260 \mathrm{~nm}$ was assigned to the dimerized moieties of the mesogenic group due to the cycloaddition

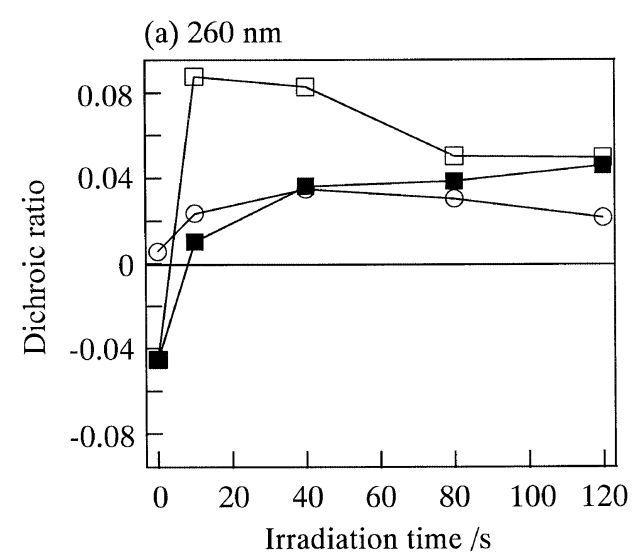

(b) $320 \mathrm{~nm}$

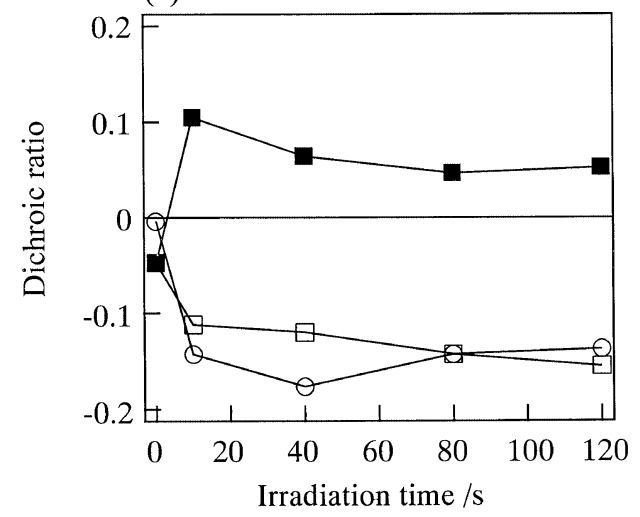

Figure 4. Dichroic ratios of the absorption peaks at $260 \mathrm{~nm}$ (a) and $320 \mathrm{~nm}$ (b) of polymer 1 film: the polymer film irradiated with linearly polarized UV light in the LC state $\left(170^{\circ} \mathrm{C}\right)$ (filled squares), after annealing (open squares) and after spin-coating (open circles).

reaction of the chalcone moiety. ${ }^{14}$ The $D R$ values of the peak at $260 \mathrm{~nm}$ were positive. This result indicated that the dimerized moieties were aligned parallel to the electric vector of the incident LPUV light.

The peak at $320 \mathrm{~nm}$ in the UV spectra of the polymer 1 film was assigned to the non-reacted chalcone derivative. The $D R$ values of the peak around $320 \mathrm{~nm}$ were dependent upon the irradiation conditions. In the case of the spin-coated and the annealed films irradiated with the LPUV light at room temperature, the sign of the $D R$ values was negative. This result showed that the non-reacted chalcone derivatives were aligned perpendicular to the vector of the incident LPUV light. On the other hand, the sign of the $D R$ values was positive for the film irradiated in the LC state. This result displayed that the non-reacted chalcone derivatives were aligned parallel to the vector of the incident LPUV light.

Kawatsuki et al. proposed that the reorientation of the non-reacted side chain containing both mesogenic and cinnamoyl groups took place during the irradiation of the LPUV light in the LC state. ${ }^{11,12}$ The authors investigated the photoreaction of the side chain type LCPs containing the cinnamoyl group. Similar 


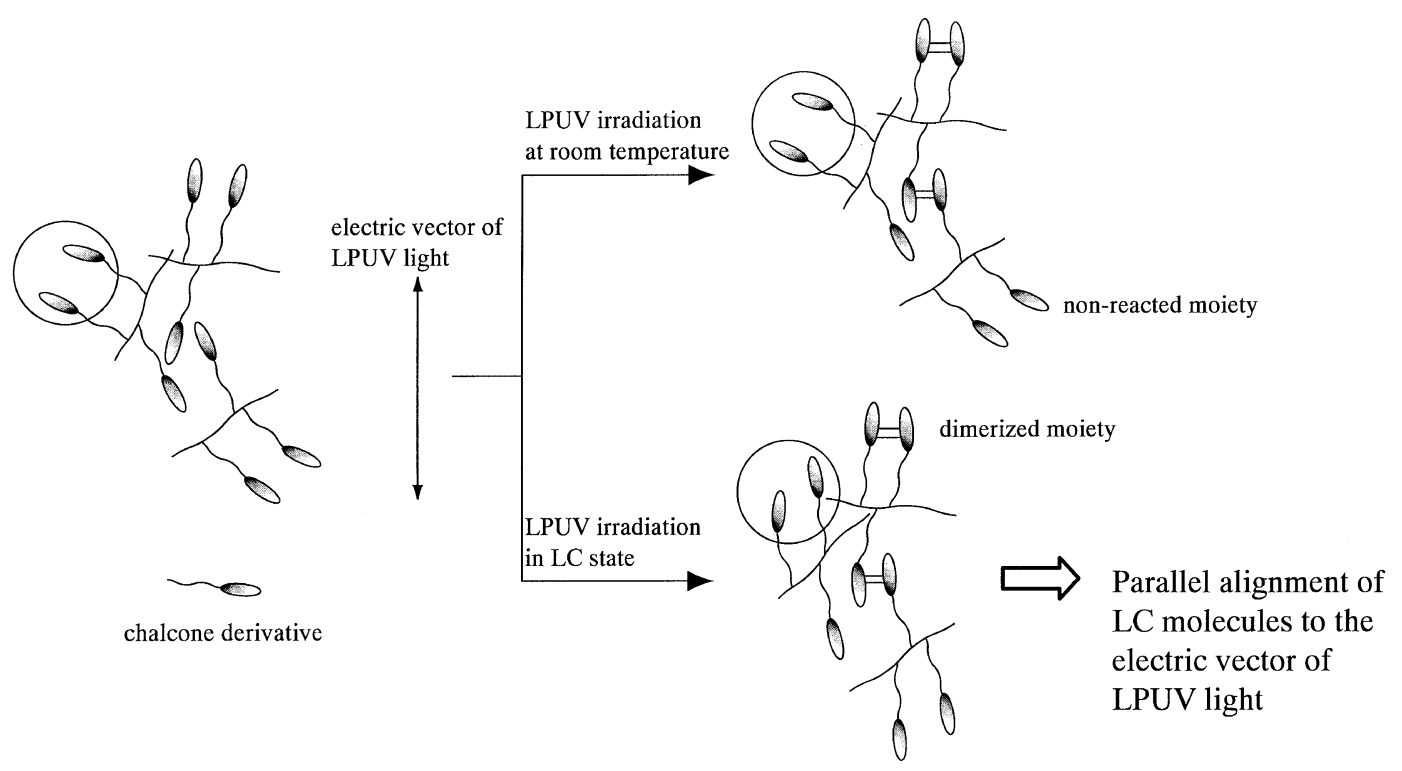

Figure 5. Schematic model of the reorientation of the non-reacted chalcone moieties in polymer $\mathbf{1}$.

reorientation behaviors of mesogenic groups containing the non-reacted chalcone derivatives were observed in our case. The photosensitive chalcone derivatives whose long axes are parallel to the electric vector of the LPUV light can react preferentially. Therefore the dimerized moieties whose long axes are parallel to the electric vector of the LPUV light would be generated. As a result, the sign of the $D R$ values of the peak at $260 \mathrm{~nm}$ was positive.

When the parallel chalcone derivatives with parallel long axis to the electric vector of the LPUV light reacted preferentially, the amount of mesogenic groups containing the non-reacted chalcone derivatives would decrease in the parallel direction to the electric vector of the LPUV light. Generally the $D R$ values of the peak at $320 \mathrm{~nm}$ would decrease and the sign of the $D R$ values would become negative. However, in the LC state film, the sign of the $D R$ values for the peak at $320 \mathrm{~nm}$ is positive. This result indicates that the non-reacted side chains would be aligned parallel to the long axis of the dimerized moieties when the dimerized moieties were generated by the LPUV irradiation. In obtaining parallel alignment of both the non-reacted and dimerized chalcone moieties, it would be necessary for the nonreacted side chains to reorient in the parallel direction to the electric vector of the incident LPUV light. The reorientation of the non-reacted side chains would be generated bya thermal motion of the non-reacted side chains in the LC phase.

On the other hand, in the case of the spin-coated and the annealed films irradiated with the LPUV at room temperature, the non-reacted chalcone derivatives cannot reorient due to their low thermal motion during the LPUV irradiation procedure at room temperature. As a result, the reorientation of the non-reacted side chains does not occur, and then the non-reacted side chains cannot be aligned parallel to the alignment direction of the dimerized moieties. A schematic model of the reorientation of the non-reacted side chain of the polymer 1 film was shown in Figure 5. In the present time, we believe that the dimerized moieties of the chalcone derivatives would play an important role in the reorientation of the non-reacted chalcone derivatives.

Figure 6 showed $D R$ values of the peaks at $260 \mathrm{~nm}$ and $300 \mathrm{~nm}$ in the polarized UV spectra of the polymer 2 films irradiated with the LPUV light under three different conditions. The peak at $260 \mathrm{~nm}$ was also assigned to the dimerized moieties of the chalconyl group. ${ }^{14}$ The $D R$ values of the peak at $260 \mathrm{~nm}$ were positive for the polymer film irradiated after spin-coating, while they were negative for the polymer films irradiated after annealing and in the LC states, respectively. They did not change with irradiation time for the polymer 2 films. These results indicated that the anisotropy based on the formation of the dimerized moieties would induce in the parallel or the perpendicular direction to the electric vector of the LPUV light. However we cannot clarify details of the induced anisotropy because the molecular shape of the dimerized moieties would have no anisotropic chemical structure compared to the nonreacted chalcone moieties.

The $D R$ values of the peak at $300 \mathrm{~nm}$ were negative for all the polymer 2 films. These results indicated that the non-reacted chalcone moieties of the polymer 2 films were aligned perpendicular to the electric vector of the incident LPUV light. The reorientation of the non-reacted chalcone moieties cannot be observed for the polymer 2 films irradiated with the LPUV light. It 
(a) $260 \mathrm{~nm}$

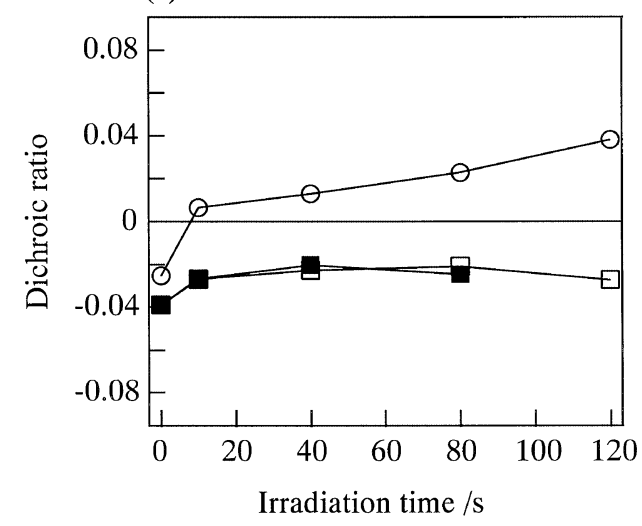

(b) $300 \mathrm{~nm}$

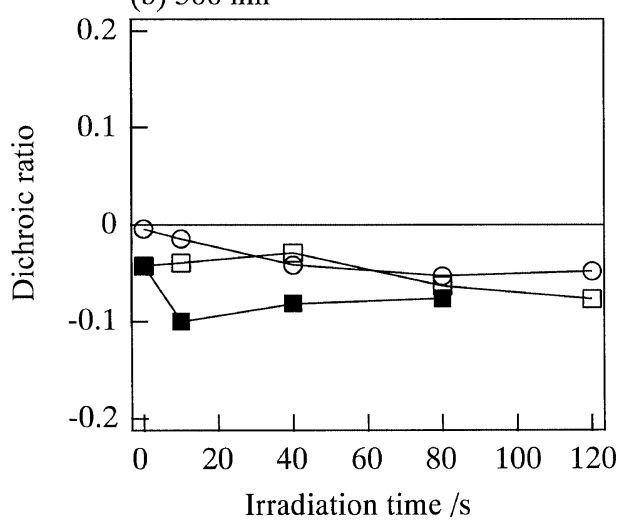

Figure 6. Dichroic ratios of the absorption peaks at $260 \mathrm{~nm}$ (a) and $300 \mathrm{~nm}$ (b) of polymer 2 film: the polymer film irradiated with linearly polarized UV light in the LC state $\left(150^{\circ} \mathrm{C}\right)$ (filled squares), after annealing (open squares) and after spin-coating (open circles).

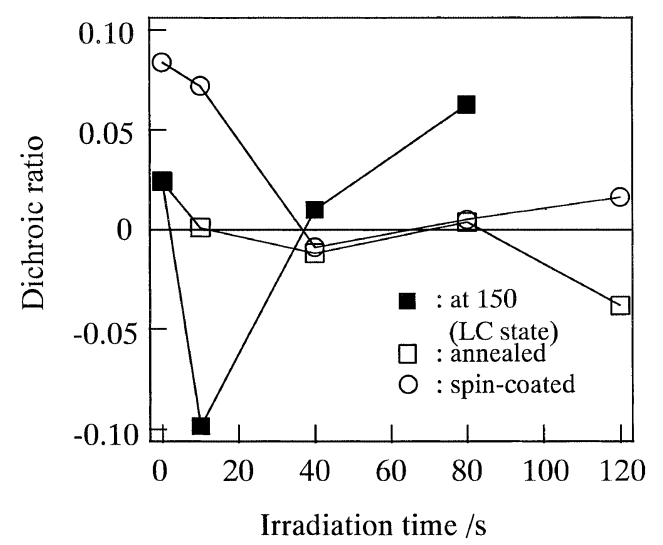

Figure 7. Dichroic ratios of the absorption peak assigned to cyano group in polymer 2 film: the polymer film irradiated with linearly polarized UV light in the LC state $\left(150^{\circ} \mathrm{C}\right)$ (filled squares), after annealing (open squares) and after spin-coating (open circles).

would be difficult to reorient for the chalcone moieties in the polymer $\mathbf{2}$ in the parallel direction to the electric vector of the LPUV light because the chalcone moieties are directly connected to the polymer backbone.

Figure 7 displayed $D R$ values of the cyanobiphenyl group in the side chain of the polymer 2 . These $D R$ values of the cyanobiphenyl group were obtained by FT-IR

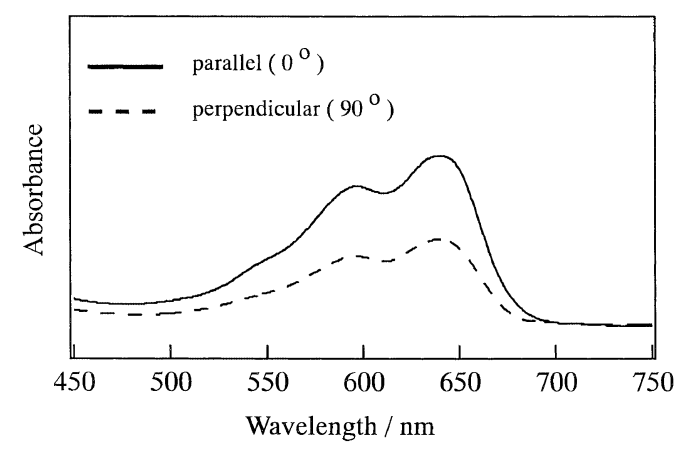

Figure 8. Polarized UV-vis spectra of the dichroic dye in liquid crystalline cell: solid and dashed lines indicate absorption spectra parallel and perpendicular to the electric vector of the incident LPUV light, respectively.

measurements of the polymer 2 films. The $D R$ values decreased with irradiation for the polymer 2 films irradiated with the LPUV light after spin-coating and annealing. This result indicated that the cyanobiphenyl groups on the surface of the polymer $\mathbf{2}$ film may be reoriented in the perpendicular direction to the electric vector of the LPUV light. The resulting absolute $D R$ values were small for the polymer $\mathbf{2}$ film irradiated after spin-coating and annealing. These results showed that finally cyanobiphenyl groups would be aligned randomly inside the polymer 2 film. However we believed that cyanobiphenyl groups on the polymer surface would be aligned perpendicular direction to the electric vector of the LPUV light on the basis of the decrease in the $D R$ at the initial state of irradiation.

The $D R$ values also decreased for the polymer $\mathbf{2}$ films irradiated in the LC state for $10 \mathrm{~s}$, however they increased with irradiation. Consequently the $D R$ values of the polymer $2 \mathrm{film}$ irradiated in the LC state were positive. This result showed that cyanobiphenyl groups both on the surface of the polymer 2 film and inside the polymer $\mathbf{2}$ film would be reoriented in the parallel direction to the electric vector of the incident LPUV light.

\section{Alignment Behavior of Low Molar Mass Liquid Crystal on Irradiated Polymer Films}

Figure 8 showed UV-vis spectra of the dichroic dye in the LC cell. We calculated the $D R$ of the dichroic dye according to the equation as shown in experimental section and investigated the alignment behavior of the LC molecules based on the $D R$. The positive $D R$ value indicated that the LC molecules were aligned parallel to the electric vector of the incident LPUV light, while the negative $D R$ value displayed that these LC molecules were aligned perpendicular to the electric vector of the LPUV light.

Figure 9 displayed an angular dependence for the ab- 


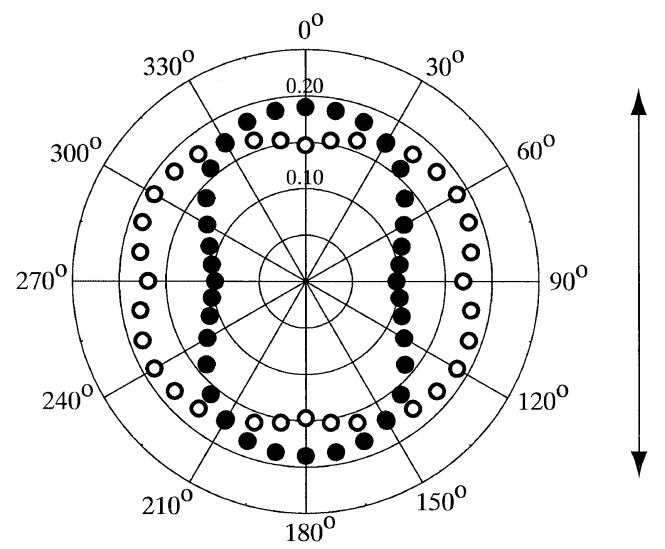

Figure 9. Circular diagram of absorbance at $640 \mathrm{~nm}$ for the dichroic dye in the liquid crystalline cell by using polymer 1 films exposed to LPUV light for $0 \mathrm{~s}$ after annealing (open circles) and 120 $\mathrm{s}$ at $170^{\circ} \mathrm{C}$ (filled circles). The arrow indicates the electric vector of the incident LPUV light.

sorption of the dichroic dye in the LC cells fabricated from polymer films irradiated with the LPUV light in LC state. Before irradiation with the LPUV light (only annealing), the absorption of the dichroic dye was independent upon the polarized angle in the polarized UVvis spectroscopy measurements. On the other hand, after irradiation with LPUV light, the absorption in the parallel direction to the electric vector of the incident LPUV light was larger than that in the perpendicular direction to the vector of the light. These results indicated that before irradiation, LC molecules were aligned randomly, and that after irradiation, LC molecules were aligned parallel to the electric vector of the incident LPUV light.

The relationship between LPUV irradiation time of the polymer films exploited for the LC cells and the $D R$ values of the LC cells were summarized in Figure 10. Positive or negative $D R$ values were detected for the LC cells using the non-irradiated $(0 \mathrm{~s})$ polymer 1 films. In the case of the spin-coated polymer 1 film, large anisotropy was not observed for the spincoated film itself as shown in Figure 4. About 0.2 of $D R$ value was observed for the $\mathrm{LC}$ cell fabricated from the spin-coated films. The $D R$ value of the LC cell fabricated from non-irradiated spin-coated films was larger than that of the LC cells fabricated from LPUV irradiated spin-coated films. The reason why larger $D R$ value was generated for the LC cell before irradiation was not clarified. The polymer surface condition of non-irradiated spin-coated film would be very complex. Very small positive $D R$ values were observed for the LC cells fabricated from the LPUV irradiated spin-coated films. These small $D R$ values indicated that no uniaxial alignment of LC molecules occurred. The alignment behavior of LC molecules was supported by polarized
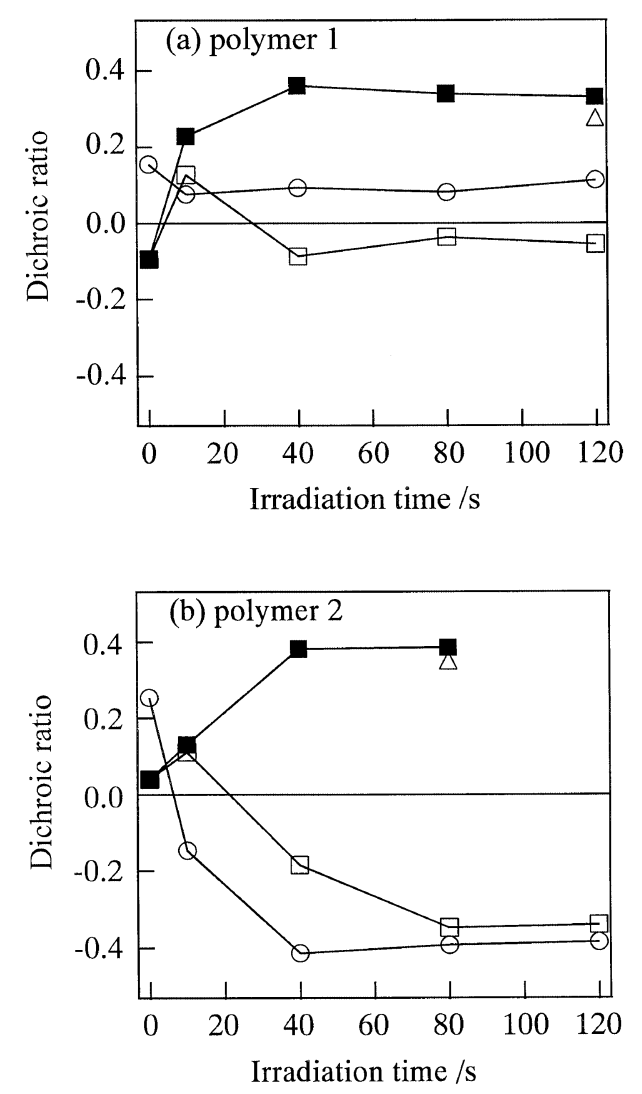

Figure 10. Dichroic ratios of the dichroic dye in the liquid crystalline (LC) cells: the LC cells fabricated from the polymer films irradiated with LPUV light in the LC state (filled squares), after annealing (open squares) and after spin-coating (open circles). Open triangles indicate the dichroic ratios of the dichroic dye in the LC cells (using polymer films irradiated with LPUV light in the LC state) after annealing.

optical microscopy measurements.

The LC cells fabricated from the annealed films also displayed small $D R$ values. This result also indicates that uniaxial alignment of the LC molecules cannot be obtained for the LC cell fabricated from the annealed films. In the case of the $\mathrm{LC}$ cell fabricated from the polymer 1 films irradiated in the LC state, the sign of the $D R$ values was positive. This result showed that the filled LC molecules were aligned parallel to the electric vector of the incident LPUV light. The uniaxial alignment behavior of the LC molecules in the LC cell was detected by polarized optical microscopy measurements. The same result was observed for the LC cells using the polymer 2 films irradiated with the LPUV light in LC state as shown in Figure 10b. These results indicated that the LC molecules were easily aligned parallel to the electric vector of the incident LPUV light in the LC cells fabricated from the polymer films irradiated in the LC state.

On the other hand, the negative $D R$ values were observed for the LC cells fabricated from the polymer $\mathbf{2}$ films irradiated with the LPUV light for 80 and $120 \mathrm{~s}$ 
after annealing, and for the LC cells using the polymer 2 films irradiated for 40, 80, and $120 \mathrm{~s}$ after spincoating. In these LC cells, the LC molecules were easily aligned perpendicular to the electric vector of the incident LPUV light. The absolute $D R$ value of the LC cell fabricated from the polymer $\mathbf{2}$ films was relatively larger compared to that for the LC cell fabricated from the polymer 1 film as shown in Figure 10b. This result showed that mesogenic groups at the end of the side chain would play an important role to exhibit relatively larger absolute $D R$ values.

As shown in Figure 10, LC molecules were easily aligned parallel to the electric vector of the incident LPUV light in the LC cell fabricated from the polymer films irradiated in the LC state, while small positive $D R$ values or negative $D R$ values were obtained for the LC cell fabricated from the spin-coated and annealed films irradiated at room temperature. As shown in Figure 4, for the polymer 1 film irradiated with the LPUV light in the LC state, the long axes of both the nonreacted chalcone derivatives and the dimerized moieties oriented in the parallel direction to the electric vector of the LPUV light. In contrast, the orientational direction of the dimerized moieties was different from that of the non-reacted chalcone derivatives for the spin-coated and the annealed films irradiated with the LPUV light at room temperature. As a result of Figures 4 and 10, the uniaxial alignment behavior of the LC molecules was achieved using the polymer 1 films where the photoinduced anisotropy would be generated by the orientation of both non-reacted side chains and dimerized moieties in the same direction.

The $D R$ values as shown in Figure 4 consisted of the information about the orientational order of the nonreacted chalcone derivatives and the dimerized moieties both on the surface of the polymer film and inside the polymer film. However the photo-induced anisotropy would reflect the anisotropy on the surface of the polymer film. The photodimerization would be propagated from the polymer surface to the bulk of polymer film. The dimerized moieties would be generated near the polymer surface, followed by the reorientation of the non-reacted moieties in the parallel direction to the electric vector of the LPUV light.

In the case of the LC cell fabricated with the spincoated and the annealed polymer $\mathbf{2}$ films irradiated with the LPUV light at room temperature, LC molecules were easily aligned perpendicular to the electric vector of the LPUV light. This alignment behavior of LC molecules in the LC cell fabricated with the spin-coated and the annealed polymer 2 films would be supported by the photo-induced anisotropy formed by the perpendicular alignment of the non-reacted chalcone moieties and the cyanobiphenyl groups on the surface of the polymer 2 film as shown in Figure 6.

In the case of the polymer 2 films irradiated with the LPUV light in the LC state, the cyanobiphenyl groups and the non-reacted chalcone moieties were aligned parallel and perpendicular to the electric vector of the LPUV light as shown in Figures 6 and 7, respectively. LC molecules in the LC cells fabricated with polymer 2 films irradiated in the LC state were aligned parallel to the electric vectors of the LPUV light as shown in Figure 10. This alignment behavior of the LC molecules would be explained on the basis of the anisotropy produced by the reorientation of the cyanobiphenyl groups. The anisotropy induced by the reorientation of the cyanobiphenyl groups would be larger than that by the alignment of the non-reacted chalcone moieties. In the case of the LC cells fabricated with the irradiated polymer 2 films, the anisotropy induced by the orientation of the cyanobiphenyl groups or the non-reacted chalcone moieties would be important for the alignment of the LC molecules in the LC cells.

We investigated thermal stability of the alignment properties for the polymer films in the LC cells. We employed the LC cells fabricated from the polymer films irradiated with the LPUV light for $120 \mathrm{~s}$ in the LC state. After each LC cell was left for $10 \mathrm{~min}$ at $200^{\circ} \mathrm{C}$ for the polymer 1 and at $170^{\circ} \mathrm{C}$ for the polymer 2 , we examined the $D R$ values at room temperature. Open triangles indicated the $D R$ values for the LC cells after annealing as shown in Figure 10. After annealing the LC cells, the $D R$ values decreased slightly, however the positive $D R$ values were obtained. These results demonstrated that the LC molecules were aligned parallel to the electric vector of the incident LPUV light after annealing. The alignment properties of the irradiated polymer films were maintained in the parallel direction to the electric vector of the incident LPUV light because the $D R$ values did not decrease remarkably and was not negative. The uniaxial alignment of the LC molecules after annealing was confirmed by polarized optical microscopy measurements.

\section{REFERENCES}

1. K. Ichimura, Chem. Rev., 100, 1847 (2000).

2. M. O’Neill and S. M. Kelly, J. Phys. D: Appl. Phys., 33, R67 (2000).

3. K. Ichimura, Y. Hayashi, H. Akiyama, T. Ikeda, and N. Ishizuki, Appl. Phys. Lett., 63, 449 (1993).

4. T. Seki, K. Ichimura, R. Fukuda, T. Tanigaki, and T. Tamaki, Macromolecules, 29, 892 (1996).

5. K. Ichimura, H. Akiyama, K. Kudo, N. Ishizuki, and S. Yamamura, Liq. Cryst., 20, 423 (1996).

6. M. Schadt, K. Scmitt, V. Kozinkov, and V. Chigrinov, Jpn. J. 
Appl. Phys., 31, 2155 (1992).

7. Y. Iimura, T. Saitoh, S. Kobayashi, and T. Hashimoto, J. Photopolym. Sci. Technol., 2, 257 (1995).

8. X. Li, D. H. Pei, S. Kobayashi, and Y. Iimura, Jpn. J. Appl. Phys., 36, L432 (1997).

9. K. Ichimura, Y. Akita, H. Akiyama, K. Kudo, and Y. Hayashi, Macromolecules, 30, 903 (1997).

10. G. P. Bryan-brown and I. C. Sage, Liq. Cryst., 20, 825 (1996).

11. N. Kawatsuki, H. Takatsuka, T. Yamamoto, and H. Ono, Jpn. J. Appl. Phys., 36, 6464 (1997).

12. N. Kawatsuki, K. Takatani, T. Yamamoto, and H. Ono, Polym. J., 30, 946 (1998).

13. Y. Makita, T. Ogawa, S. Kimura, M. Nakata, S. Kimura, Y. Matsuki, and Y. Takeuchi, IDW '97, FMC3-3, 363 (1997).

14. Y. Makita, T. Natsui, S. Kimura, S. Nakata, M. Kimura, Y. Matsuki, and Y. Takeuchi, J. Photopolym. Sci. Technol., 11, 187 (1998).
15. Y. Makita, T. Natsui, S. Kimura, M. Nakata, S. Kimura, Y. Matsuki, and Y. Takeuchi, SID 98 Digest, LP-H, 750 (1998).

16. S. Nakata, K. Kuriyama, M. Kimura, T. Natsui, Y. Makita, Y. Matsuki, N. Bessho, and Y. Takeuchi, SID 98 Digest, LP-3, 512 (1999).

17. D.-S. Seo and J.-Y. Hwang, Jpn. J. Appl. Phys., 39, L816 (2000).

18. D.-S. Seo and J.-Y. Hwang, Jpn. J. Appl. Phys., 39, L1239 (2000).

19. M. Kimura, S. Nakata, Y. Makita, Y. Matsuki, A. Kumano, Y. Takeuchi, and H. Yokoyama, Jpn. J. Appl. Phys., 40, L352 (2001).

20. Y. Iimura, T. Saito, S. Kobayashi, and T. Hashimoto, J. Photopolym. Sci. Technol., 8, 257 (1995).

21. T. Mihara, M. Tsutsumi, and N. Koide, Mol. Cryst. Liq. Cryst., in press. 